Autofluorescence dynamics during reperfusion following long-term renal ischemia in a rat model

R. N. Raman, C. D. Pivetti, D. L. Matthews, C.

Troppmann, S. G. Demos

February 14,2008

Photonics West

San Jose, CA, United States

January 18, 2008 through January 25, 2008 
This document was prepared as an account of work sponsored by an agency of the United States government. Neither the United States government nor Lawrence Livermore National Security, LLC, nor any of their employees makes any warranty, expressed or implied, or assumes any legal liability or responsibility for the accuracy, completeness, or usefulness of any information, apparatus, product, or process disclosed, or represents that its use would not infringe privately owned rights. Reference herein to any specific commercial product, process, or service by trade name, trademark, manufacturer, or otherwise does not necessarily constitute or imply its endorsement, recommendation, or favoring by the United States government or Lawrence Livermore National Security, LLC. The views and opinions of authors expressed herein do not necessarily state or reflect those of the United States government or Lawrence Livermore National Security, LLC, and shall not be used for advertising or product endorsement purposes. 


\title{
Autofluorescence dynamics during reperfusion following long-term renal ischemia in a rat model
}

\author{
Rajesh N. Raman ${ }^{\mathrm{a}}$, Christopher D. Pivetti ${ }^{\mathrm{b}}$, Dennis L. Matthews ${ }^{\mathrm{a}}$, \\ Christoph Troppmann ${ }^{\mathrm{b}}$, Stavros G. Demos ${ }^{\mathrm{c}}$ \\ ${ }^{a}$ University of California, Davis, Dept. of Applied Science, Davis, CA 95616 \\ ${ }^{\mathrm{b}}$ University of California, Davis Medical Center, Dept. of Surgery, Sacramento, CA 95817 \\ ${ }^{c}$ Lawrence-Livermore National Laboratory, Livermore, CA 94550 and University of California, \\ Davis Medical Center, Dept. of Urology, Sacramento, CA 95817
}

\begin{abstract}
Optical properties of near-surface kidney tissue were monitored in order to assess response during reperfusion to long (20 minutes) versus prolonged (150 minutes) ischemia in an in vivo rat model. Specifically, autofluorescence images of the exposed surfaces of both the normal and the ischemic kidneys were acquired during both injury and reperfusion alternately under $355 \mathrm{~nm}$ and $266 \mathrm{~nm}$ excitations. The temporal profile of the emission of the injured kidney during the reperfusion phase under $355 \mathrm{~nm}$ excitation was normalized to that under $266 \mathrm{~nm}$ as a means to account for changes in tissue optical properties independent of ischemia as well as changes in the illumination/collection geometrical parameters in future clinical implementation of this technique using a hand-held probe. The scattered excitation light signal was also evaluated as a reference signal and found to be inadequate. Characteristic time constants were extracted using fit to a relaxation model and found to have larger mean values following 150 minutes of injury. The mean values were then compared with the outcome of a chronic survival study where the control kidney had been removed. Rat kidneys exhibiting longer time constants were much more likely to fail. This may lead to a method to assess kidney viability and predict its ability to recover in the initial period following transplantation or resuscitation.
\end{abstract}

Keywords: in vivo, ischemia, kidney, autofluorescence, organ transplantation, rat, reperfusion, tissue viability

\section{INTRODUCTION}

Patients with candidate transplant tissues that have undergone an unknown yet significant degree of ischemia are commonly encountered at the transplant clinic. During ischemia, affected tissue is deprived of oxygen and nutrients supplied by the blood that are necessary to conduct normal cellular functions, such as maintenance of ion gradients or synthesis of important complex biomolecules. Early tissue changes induced by ischemia include cellular swelling and leakage which are difficult to quantify, even with standard histopathology using stained tissue specimens. Microdialysis has been used to monitor the concentration of extracellular metabolism-related molecules such as $\mathrm{H}+$ and lactate in vivo $^{1-2}$. Probe insertion itself, however, can alter local tissue hemodynamics and metabolism ${ }^{3}$. A noninvasive method is needed when handling tissue whose functional status is critical or unknown.

The advantages of optical spectroscopy methods for tissue characterization are well recognized and include the ability to detect biochemical changes, with the short acquisition times necessary for real-time in vivo analysis. Specific optical measurements of changes in cell metabolism were first made by Chance ${ }^{4}$ by monitoring the autofluorescence of $\mathrm{NADH}$, an intrinsic fluorescing biomolecule whose concentration is sensitive to hypoxic conditions. Since then, studies have primarily examined short-term ischemia (over several minutes) that is detected locally, where fiber optic microfluorometers typically illuminate a spot less than $2 \mathrm{~mm}$ in diameter ${ }^{5-8}$.

However, in our approach, we are concerned with monitoring the following parameters with clinical in vivo evaluation in mind: 1) longer periods of ischemia, for example tens of minutes to hours, 2) heterogeneous tissue response over a region larger than that previously monitored, 3) dynamic tissue response during the reperfusion phase, when the cells are given the first opportunity to recover, and 4) scattering properties of the tissue that can change due to tissue dehydration, movement during in vivo measurements, and probe-to-tissue geometry during future implementation of this technique in the clinic. Regarding this last point, in our previous results we found that by monitoring extended periods of ischemia over the full kidney surface in an imaging arrangement and using the contralateral (normal) kidney as a control, the intensity from the injured kidney under $335 \mathrm{~nm}$ excitation was sensitive to ischemia and reperfusion, while that under $260 \mathrm{~nm}$ excitation was not sensitive to either ischemia or reperfusion (Fig. 1) ${ }^{9}$. Normalization of the 
ischemia-sensitive signal to that which is insensitive may provide a means to account for changes in tissue scattering properties not dependent on the ischemia process.

In this work we evaluate the ability of the autofluorescence signal ratio method to 1) differentiate kidneys having undergone long ischemic injury (20 $\mathrm{min}$ ) from those having undergone prolonged injury (150 min), and 2) predict which kidneys would fail, based on a model quantifying emission dynamics during reperfusion. The model took the form of a double exponential function in order to take into account changes in autofluorescence in addition to those due to a change in NADH concentration. We postulate that a correlation between the results of this optical signal ratio analysis and post-reperfusion kidney function may lead to optical methods to predict organ response following transplantation or resuscitation.

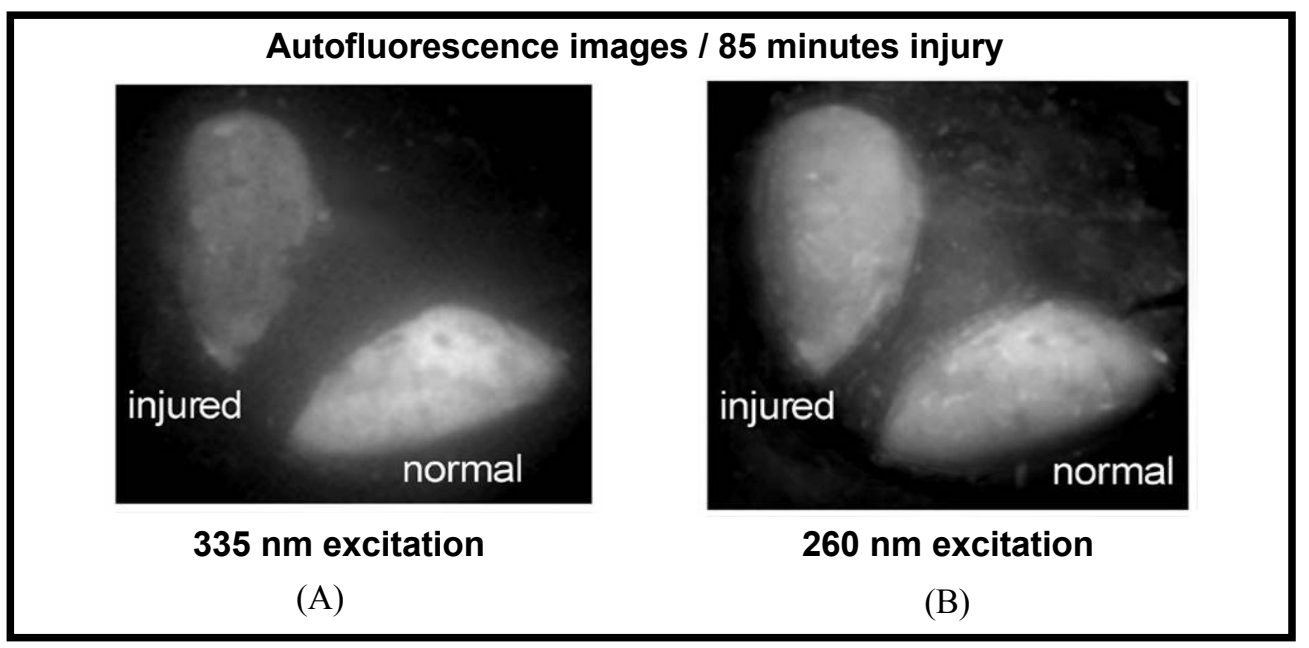

Figure 1. In vivo comparison of autofluorescence intensity of injured kidney after 85 minutes of ischemia under $335 \mathrm{~nm}(\mathrm{~A})$ and $260 \mathrm{~nm}$ (B) excitations.

\section{METHODS}

\subsection{Rat preparation and renal ischemia}

All animal procedures were approved by the University of California, Davis, Animal Use and Care Administrative Advisory Committee (AUCAAC). Adult male Spontaneously Hypertensive Rats (SHR) aged 16-17 weeks and weighing 350-400 g were allowed free access to standard pellet chow and tap water. Rats were placed under general anesthesia using $2 \%$ isoflurane delivered with $1.0 \mathrm{~L}$ of air per minute. Depth of anesthesia was monitored by toe pinch tests given at 15 minute intervals during the procedure. Exhaled gases were disposed of appropriately using a filter and scavenging system. Following anesthesia induction, the animal's chest and abdomen were shaved and prepared with alternating rounds of iodine scrub and $70 \%$ ethanol. Next, a midline laparotomy was performed, the small bowel exteriorized, and the left renal pedicle exposed. A non-fluorescent black cloth was then placed over the surrounding tissue to prevent nonkidney tissue autofluorescence. In order to keep the abdominal cavity moist, the intestine was wrapped in saline-soaked gauze, and saline was dripped on the kidneys at 5 minute intervals. During the monitoring period, the rat was kept warm on a heating pad.

A bulldog clamp was used to occlude arterial and venous flow resulting in unilateral ischemic injury to the left kidney, henceforth referred to as the injured kidney. The contralateral right kidney was undisturbed. Ischemia was induced for either $20(\mathrm{n}=9$ rats) or $150(\mathrm{n}=10)$ minutes. At the end of the injury phase, the clamp was released and the kidneys were allowed to reperfuse. Following reperfusion (at least $45 \mathrm{~min}$ following a $20 \mathrm{~min}$ injury, and at least $60 \mathrm{~min}$ following a 150 min injury), the normal kidney was removed by means of a contralateral nephrectomy. Postoperatively, rats were given $0.3 \mathrm{cc}$ Buprenex pain medication (Henry Schein, Melville, NY). Rat weight, physical appearance, and survival were monitored for 30 days following ischemia, or until rat lost more than $20 \%$ of preoperative body weight (at which time it was sacrificed by exsanguination), or until death. 


\subsection{Optical spectroscopy methods}

A schematic layout of the experimental arrangement is shown in Fig. 2. Two separate laser sources were utilized in order to alternately illuminate the kidneys with two excitation wavelengths in real-time. Specifically, two compact diode-pumped solid state (DPSS) lasers were used operating at $355 \mathrm{~nm}$ (Meshtel, Genoa, NV) and $266 \mathrm{~nm}$ (Power Technology, Inc., Little Rock, AR), both operating at $1 \mathrm{kHz}$ repetition rate with $5 \mu \mathrm{J} /$ pulse. Separate shutters (NM Laser Products, Inc., Sunnyvale, CA) were positioned at the output of each laser (Fig. 2), each with a $5 \mathrm{~mm}$ aperture. These shutters were controlled electronically by a PC using Labview software (National Instruments, Austin, TX). The two beam paths were arranged to co-propagate using dielectric mirrors and were coupled into a fiber bundle (Ocean Optics, Dunedin, FL) which delivered the excitation light to the tissue at a dosage of $0.22 \mathrm{~mJ} / \mathrm{cm}^{2}$. The fiber output, located 45 $\mathrm{cm}$ from the tissue, was allowed to expand to fully illuminate both kidneys. The autofluorescence images were recorded through a 420-640 $\mathrm{nm}$ band-pass filter positioned in front of a liquid nitrogen-cooled CCD camera (Roper Scientific, Trenton, NJ). A quartz slide was placed at $45^{\circ}$ to reflect some of the signal to a second CCD (Roper Scientific) in order to monitor the scattered excitation light. A UV-pass filter rejected the autofluorescence portion of this signal entering the second camera. A baseline image was acquired $(\mathrm{t}=0)$ before the clamp was applied, then imaging continued immediately after the clamping. Image exposure time was 4 seconds, taken at 15 second intervals over the course of the injury and reperfusion phases.

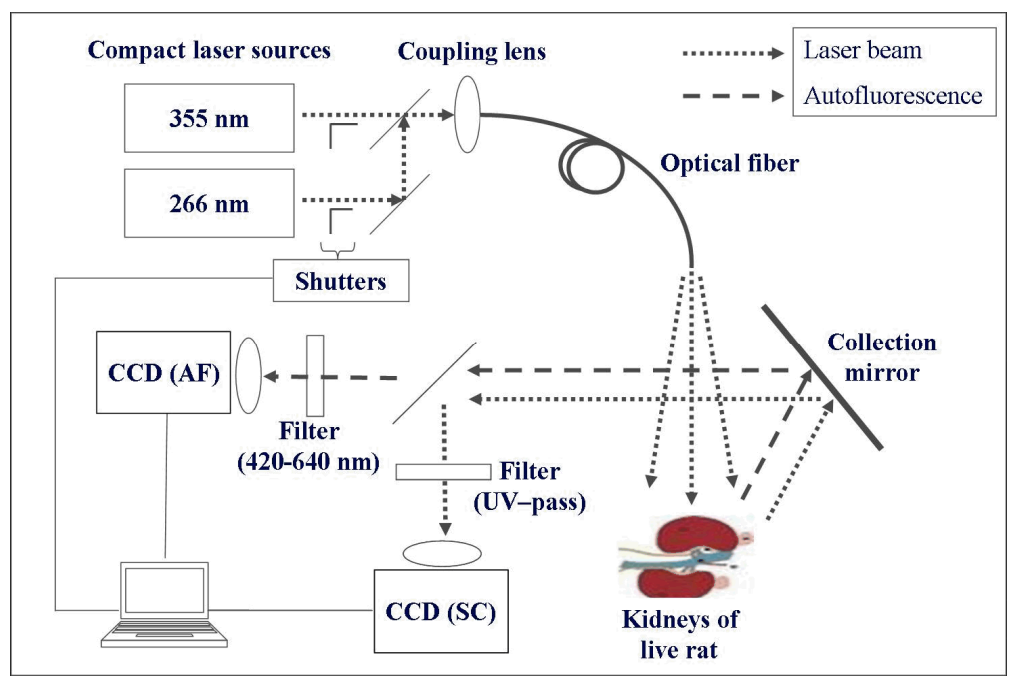

Figure 2. Autofluorescence imaging arrangement with dual wavelength excitation. AF signifies autofluorescence and SC signifies excitation light scattering.

Offline, each image was normalized to the beam spatial profile as recorded on a homogeneous fluorescent piece of paper at the start of the experiment. In addition each image was normalized to laser power as recorded by a small fluorescent piece of plastic placed alongside the kidneys. Average intensities over as large as possible an area $(\sim 1.0$ $\mathrm{cm}^{2}$ ) were recorded for each kidney surface under each excitation wavelength. The region of the tissue exhibiting specular reflection was avoided during analysis since the scattered excitation was also being monitored. The ratio formed by the emission intensity of the injured kidney under $355 \mathrm{~nm}$ excitation to that under $266 \mathrm{~nm}$ excitation was calculated (henceforth referred to as the "signal ratio").

\section{RESULTS}

\subsection{Signal under UV excitation}

The scattered excitation light has traditionally been used to monitor the change in scattering properties of the tissue during hypoxia that influence the detected autofluorescence intensity ${ }^{6}$. In the clinic it is important to keep the exposed tissue moist during surgical procedures, so in all of our experiments we applied saline to each kidney surface every 5 min. Fig. 3 compares the scattered signal to the autofluorescence signal under $355 \mathrm{~nm}$ excitation during the last hour of injury in one rat that underwent a total of $150 \mathrm{~min}$ of injury that underwent a total of $150 \mathrm{~min}$ of injury. In this period late into injury, no dynamics of the autofluorescence signal was observed, but a large change in the scattered signal 
occurred when saline was applied. On average a 7\% increase in the scattered intensity was observed each time saline was applied, followed by a decline as the water was absorbed into the tissue or evaporated until the next drop was applied.

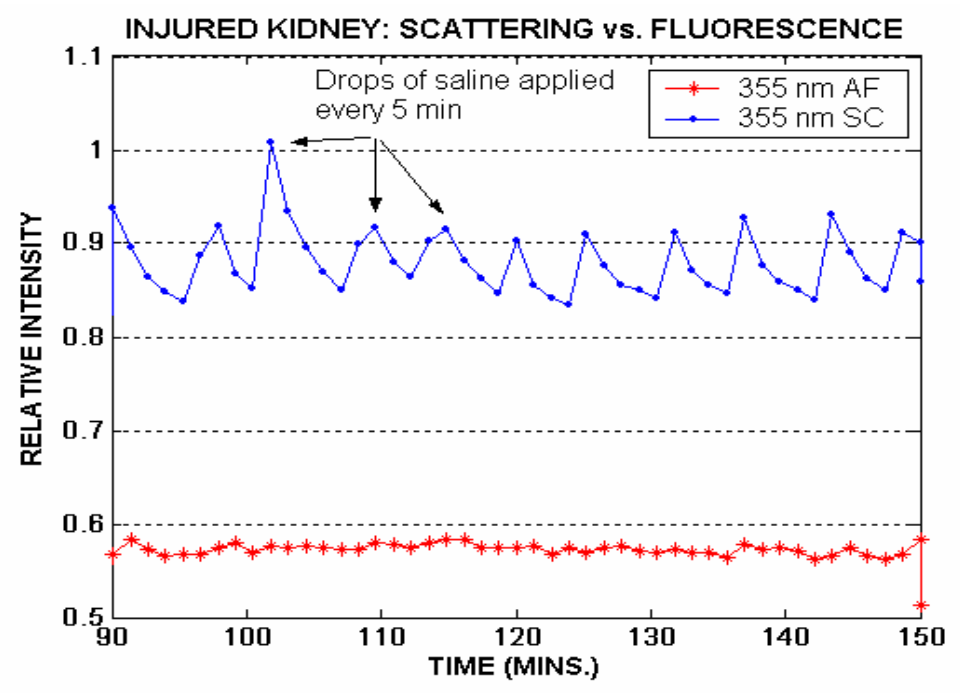

Figure 3. Scattered (SC, blue circles) vs. fluorescence signal (AF, red stars) under $355 \mathrm{~nm}$ excitation showing that the scattered excitation is sensitive to tissue hydration state.

Figure 4 (A) shows a typical emission intensity ratio profile during 150 minutes of injury and 90 minutes of reperfusion. The signal ratio at $\mathrm{t}=0$ is normalized to the pre-injury value of 1 . The letters $(\mathrm{a}, \mathrm{b}, \mathrm{c})$ represent characteristic features in the profile. Upon clamping, the signal ratio in this case decreased by almost $30 \%$ until point (a), after which the ratio did not change dramatically. After unclamping, the signal ratio increased in two phases. Initially it increased rapidly from point (b) to its first peak (c), followed by a decrease and an inflection (which was more pronounced for the case of longer injury), then finally increasing more slowly toward its final value.

\subsection{Signal ratio analysis with double exponential function}

The experimental results indicate that upon clamping, the signal decreased overall during the injury phase. Since NADH concentration increases during oxygen deprivation ${ }^{10}$, the observed decrease cannot be attributed to the increase in the amount of NADH but rather some other process modulating the NADH emission. To account for other possible contributions to the signal profile separate from a change in NADH concentration, such as that due to a change in NADH emission yield or changes in the optical properties of the tissue, we explored the possibility that the signal may be modeled by a double exponential function. The first exponential can approximate the increase in NADH, while the second exponential may represent a change in the NADH emission intensity due to the environment. During the reperfusion phase, the two exponential components take the following forms:

$$
\begin{aligned}
& \text { Component 1: } \mathrm{R}_{\mathrm{N}}= \begin{cases}\mathrm{R}_{\mathrm{N} 0} & \mathrm{t}_{\mathrm{r}}<\mathrm{t}<\Delta \tau \\
\mathrm{R}_{\mathrm{N} 0}-\Delta \mathrm{R}_{\mathrm{N}} *\left(1-\operatorname{Exp}\left(-(\mathrm{t}-\Delta \tau) / \tau_{\mathrm{N}}\right)\right) & \mathrm{t}>\Delta \tau\end{cases} \\
& \text { Component 2: } \mathrm{R}_{\mathrm{E}}= \\
& \mathrm{R}_{\mathrm{E} 0}+\Delta \mathrm{R}_{\mathrm{E}} *\left(1-\operatorname{Exp}\left(-\mathrm{t} / \tau_{\mathrm{E}}\right)\right)
\end{aligned}
$$

$R_{N}$ and $R_{E}$ represent the contribution of each component to the measured signal ratio. The intensity of the first component decreases exponentially (with relaxation time $\tau_{\mathrm{N}}$ ) by the amount $\Delta \mathrm{R}_{\mathrm{N}}$ after a delay $\Delta \tau$, while the intensity of the second component increases exponentially (with relaxation time $\tau_{\mathrm{E}}$ ) by the amount $\Delta \mathrm{R}_{\mathrm{E}}$ at the onset of reperfusion when the clamp is released. Time $t$ is measured in reference to this onset of reperfusion $\left(t_{\mathrm{r}}\right)$, and $\mathrm{R}_{\mathrm{N} 0}$ and $\mathrm{R}_{\mathrm{E} 0}$ are the initial values of components 1 and 2, respectively, at the start of reperfusion. The resultant model is formed by the product of these two components and satisfies the following conditions: the product $\mathrm{R}_{\mathrm{N} 0} * \mathrm{R}_{\mathrm{E} 0}$ is set equal to the initial 
measured signal ratio value, $\Delta \tau$ represents the delay time after the clamp is released until the signal rises to its first peak (Fig. 4(A), point (c)), and the signal ratio asymptote long after unclamping is measured and set equal to the product $\left(\mathrm{R}_{\mathrm{N} 0}\right.$ $\left.-\Delta \mathrm{R}_{\mathrm{N}}\right) *\left(\mathrm{R}_{\mathrm{E} 0}+\Delta \mathrm{R}_{\mathrm{E}}\right)$.

Signal dynamics is described by the relaxation parameter for each component $\left(\tau_{\mathrm{N}}\right.$ and $\left.\tau_{\mathrm{E}}\right)$ and the delay parameter $\Delta \tau$ for the first component. An example data fit and signal decomposition during reperfusion preceded by 20 minutes of injury is provided in Fig. 4(B). Best-fit values for $\tau_{\mathrm{N}}, \tau_{\mathrm{E}}$, and $\Delta \tau$ are labeled. Fits to the measured signal ratio intensity vs. time were performed with custom code using MATLAB software (The Mathworks, Natick, MA) by adjusting the two relaxation times $\tau_{\mathrm{N}}$ and $\tau_{\mathrm{E}}$ as well as each component's initial value $\mathrm{R}_{\mathrm{N} 0}$ and $\mathrm{R}_{\mathrm{E} 0}$. Best-fit values were achieved when the mean absolute error between resultant model and measured data was minimized.

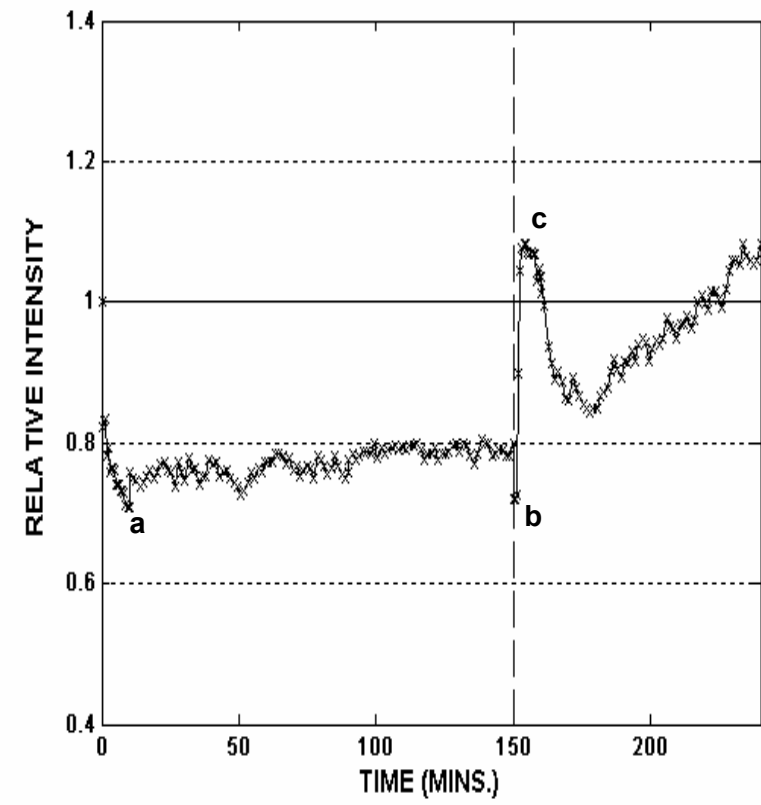

(A)

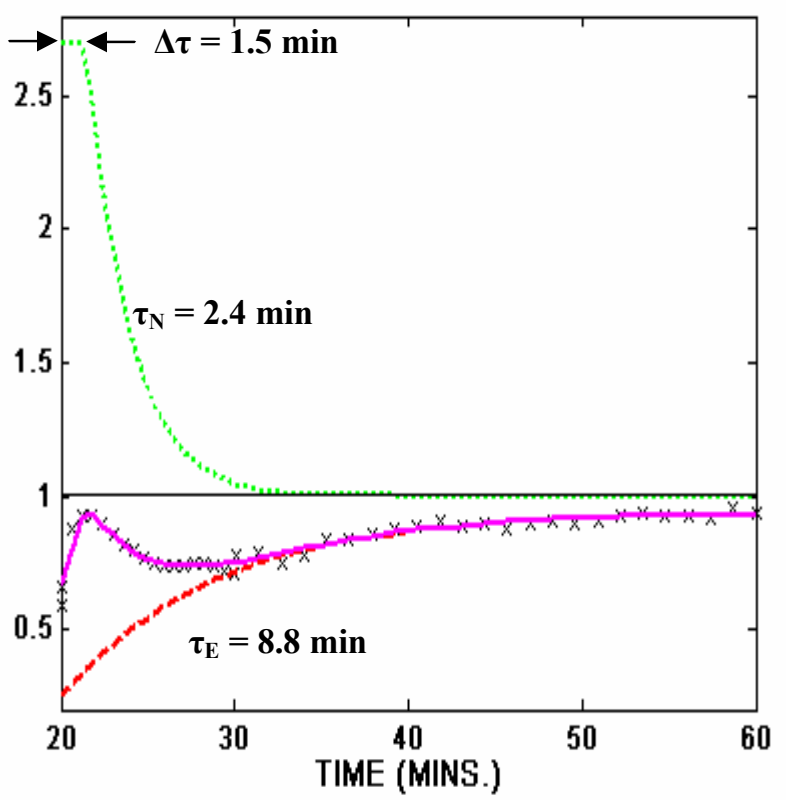

(B)

Figure 4. (A) A typical signal ratio profile from a rat kidney exposed to $150 \mathrm{~min}$. of injury. The letters (a,b,c) represent characteristic features in the real-time signal ratio. B) An example fit of the double-exponential model to the signal ratio from the reperfusion phase of a rat exposed to $20 \mathrm{~min}$ ischemia, and the extracted time constants. Dotted-green: component 1. Dashed-red: component 2. Solid magenta: product of components 1 and 2 and best-fit to data, which is shown as x's.

The first component is shown in the dotted green trace which decreased exponentially after a delay, and the second component is shown in the dashed red trace which increased to a maximum value. Figure 5 shows the results of two more fits based on this analysis. In this figure, a comparison is made between the results of analysis for a kidney exposed to 20 minutes of ischemia against that of a kidney exposed to 150 minutes of ischemia. Included with each case are two white light images of the rat kidneys in vivo, analogous to what a surgeon would observe under room light. The first image in each row was taken at the conclusion of the injury phase of the specified duration, and the second was taken 10 minutes after the clamp was released. Injured kidneys in frames a) and b), resembled each other in color and intensity despite undergoing different injury times. A similar observation is made in frames c) and d) after blood flow had been restored. Not only do the two injured kidneys resemble each other, they resemble their corresponding control kidneys. Alongside the images are the values of the time constants extracted from fits to the double exponential model. The longer injury time yielded longer time constants $\Delta \tau, \tau_{\mathrm{N}}$, and $\tau_{\mathrm{E}}$. The rat that received the 150 min injury died 5 days following the removal of the normal kidney, whereas the rat that received the 20 min injury survived the month-long observation following normal kidney removal. 


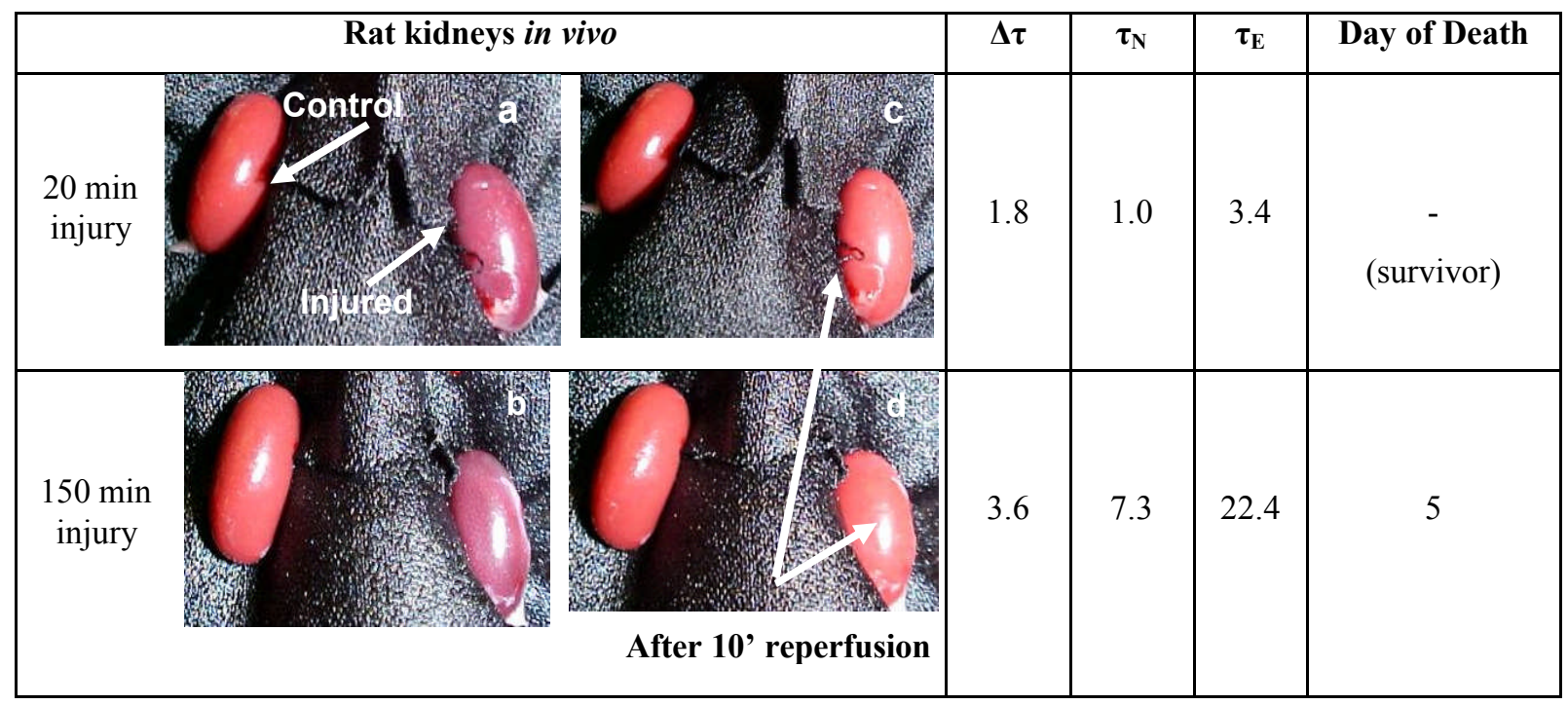

Figure 5. Comparison of white light images of kidneys from two rats that have undergone different periods of ischemia, with corresponding time constants. Injured kidneys in frames a) and b) are shown following 20 minutes and 150 minutes of ischemia, respectively. Injured kidneys in frames c) and d) are shown after 10 minutes of reperfusion. Time constants were extracted from fits to the signal ratio temporal profile.

\section{DISCUSSION}

Tissue changes induced by ischemia include cellular and organellular swelling and leakage which are difficult to quantify in the clinic. Currently no tool is available in the transplant clinic for surgeons to measure the degree of ischemic injury incurred in tissue or to monitor the return of tissue function following restoration of blood flow. Existing technology for assessing the degree of ischemia is quite invasive. Histopathology requires cutting and removal of a segment of the suspected tissue, followed by fixing, staining, and lab analysis. Microdialysis is a real-time technique but requires the insertion of a $1 \mathrm{~mm}$-diameter needle into the tissue to sample the degradative products of ischemia in the extracellular environment ${ }^{11-12}$. When dealing with tissue of critical or unknown functional status, a noninvasive method is needed.

Optical spectroscopy is a noninvasive technique that has been used for its diagnostic capabilities in probing the degree of tissue ischemic injury. For example, near-infrared spectroscopy has been used to measure tissue oxygen saturation ${ }^{13-14}$ as a means of inferring tissue functional status. But visual observation of the tissue is the most common method of assessing the degree of ischemic injury. This method is qualitative and therefore highly subjective. In addition, assessment under the red and near-infrared spectral regions is governed largely by the presence of hemoglobin and its oxygenation state. Such observations depend on properties of blood, such as the quality of perfusion and state of oxygenation, and not on cellular activity. Unfortunately, the only reliable way to assess the degree of ischemic injury is to transplant the organ and see if it works.

A more direct probe of tissue function than blood distribution is cell metabolic state. We are interested in NADH, a biomolecule located inside the cells, for two reasons. First, NADH participates directly in cellular metabolism in the process of oxidative phosphorylation. In the presence of oxygen, the energy associated with the reduction of NADH to its oxidized state $\mathrm{NAD}^{+}$is stored in ATP to drive various cellular functions. This process is reversible, and in the absence of oxygen (as in the case of ischemia) NADH can no longer be oxidized aerobically and NADH concentration increases in the cells. Second, the reduced form NADH fluoresces under UV excitation, while its oxidized form does not. NADH fluorescence under UV excitation has been observed to increase during the first several minutes of animal hypoxia ${ }^{5,15}$. A similar increase in autofluorescence signal has been observed during ischemia of the kidney, followed by a decrease towards baseline during reperfusion ${ }^{8}$.

In our approach we are concerned with several clinically relevant situations. First, we monitored long-term ischemia and reperfusion (on the order of tens of minutes to hours) since such significant durations are a clinical reality. Second, heterogeneous tissue response to ischemia and reperfusion was taken into account by imaging the entire exposed 
surface of the kidney, a departure from what has been done in the past with microfluorometer-assisted measurements. Those measurements are typically made with optical fibers in contact with less than a $2 \mathrm{~mm}$-diameter region of tissue and may be dependent on local tissue structure, such as the presence of vasculature. We then averaged the intensity over as large an area of the kidney as possible to generate the signal profile shown in Fig. 3(A). Third, we investigated the tissue response in particular during reperfusion, when the tissue may exhibit its ability to physiologically recover from ischemia and provide optical signatures permitting the early prediction of tissue recovery. Fourth, we calculated the ratio of emission under $355 \mathrm{~nm}$ to that under $266 \mathrm{~nm}$ in order to account for changes in 1) tissue scattering properties and 2) probe-tissue geometry during future clinical implementation.

Conventional normalization of the NADH autofluorescence signal incorporates the reflected $355 \mathrm{~nm}$ excitation to account for changes in blood volume that influence the NADH emission intensity ${ }^{6}$. In Fig. 3, both the autofluorescence and scattered excitation light were monitored in our experiments. The scattered signal (blue circles) was found to be very sensitive to the hydration level of the tissue and is therefore not a reliable normalization parameter. A varying hydration state can occur clinically as the tissue dehydrates during extended monitoring periods when the organs are exposed and when the surgeon rehydrates the tissue with saline to keep it moist. In addition, movement artifacts and changes in probe-to-tissue geometry can influence the scattering intensity. To account for intensity changes not associated with ischemia we coupled a secondary excitation beam into the same fiber as that of the primary excitation, which illuminates the tissue with the same geometry as that of the primary excitation. The wavelength was chosen to excite a fluorophore whose emission is independent of ischemia. We chose tryptophan because it is an amino acid and is not directly involved in cell metabolism. Tryptophan contains a broad absorption band around $280 \mathrm{~nm}$. Based on our previous findings that the visible emission under $260 \mathrm{~nm}$ excitation is insensitive to ischemia and reperfusion (Fig. 1(B)), ${ }^{9}$ a secondary source that excites tryptophan (commercially available at $266 \mathrm{~nm}$ in a compact form) was employed.

Thus the signal ratio was calculated and observed to exhibit differences in features during reperfusion for the different injury cases (Fig. 4). Specifically, the time required for the ratio to reach its initial peak (point (c)) occurred later in the 150-min injury case, and the inflection that followed was more pronounced for the longer injury. These differences were quantified by the adoption of the double exponential model described in Section 3.2. In the analysis of the experimental results presented in the previous section, the first exponential component may be attributed to the contribution of NADH concentration to the signal which decreases upon reperfusion as NADH gets oxidized due to availability of oxygen. In general, the measured fluorescence intensity from tissue depends not only on fluorophore concentration but also on its environment. Thus, the second exponential component describes the modulation of the NADH emission intensity by the environment that causes a decrease in the signal during injury and an increase during reperfusion. One possible cause is that the emission yield of NADH is being reduced during injury, caused by either the increasing level of acidity in the cells or some other by-product of ischemia. For example a change in $\mathrm{pH}$ is known to alter fluorophore emission efficiency, ${ }^{16}$ and a decrease in $\mathrm{pH}$ indeed occurs in the tissue during ischemia. ${ }^{8} \mathrm{An}$ exponential form was assumed for each component in order to model the asymptotic behavior of the resultant signal observed in both the injury and reperfusion phases and can be described by a single relaxation time parameter.

The exponential forms in Eqns. 1 and 2 have the following features during the reperfusion phase. They are described by the time constants $\tau_{\mathrm{N}}$ and $\tau_{\mathrm{E}}$, the relaxation times of the components representing the contribution of NADH concentration and NADH emission modulation, respectively. A delay time $\Delta \tau$ is introduced in the first component and may be expected, depending on the severity of the injury, because of the spatial separation between the capillary carrying the oxygenated blood and the site of electron transport in the double-membrane intracellular mitochondria and which may retard $\mathrm{O}_{2}$ delivery to the electron transport chain. An example signal ratio decomposition and data fit during reperfusion preceded by 20 minutes of ischemia is displayed in Fig. 4(B). Best-fit values for $\tau_{\mathrm{N}}, \tau_{\mathrm{E}}$, and $\Delta \tau$ are labeled.

Figure 5 displays the results of fits for a 20 min injury case versus a 150 min injury case and corresponding white light images indicating what would be seen by visual observation alone. Under white light the injured kidneys both appear dark-red in color at the conclusion of injury, regardless of injury time. Similarly after 10 minutes of reperfusion, the injured kidneys share the same bright red color as each other and as the controls. To verify that the kidney that underwent $150 \mathrm{~min}$ of injury was injured to a greater degree, rat survival was monitored. The rat subjected to $150 \mathrm{~min}$ ischemia died 5 days after the insult from renal failure (based on observations of weight and general appearance, in conjunction with the absence of confounding factors during animal autopsy). Visual observation in these cases does not distinguish the two kidneys based on injury time or function. However, fits of the double exponential model to the temporal profile of the signal ratio exhibited distinct values for time constants in the two cases. The longer, eventually fatal injury yielded longer time constants within 90 min of reperfusion.

The temporal signal ratio profile of the injured kidney exhibited a return towards baseline during reperfusion and therefore exhibited (perhaps partially) reversible optical properties. The trend that longer injuries yielded longer time 
constants during reperfusion suggests that the time for reversal of tissue optical properties increases with the severity of the injury. Indeed microdialysis measurements show that increases in metabolic products in the tissue environment caused by ischemia can be reversible during reperfusion. ${ }^{12,19}$ A potential application of this method would be to determine the characteristic time constants for a tissue recently transplanted or resuscitated and use these values to assist in the determination of tissue viability.

\section{CONCLUSIONS}

The ratio of autofluorescence intensities under $355 \mathrm{~nm}$ and $266 \mathrm{~nm}$ excitations is a technically feasible normalization procedure for the implementation of this method in a clinical setting. The scattered excitation light at 355 $\mathrm{nm}$ depends on tissue hydration level. The autofluorescence from the signal ratio is sensitive to the course of renal ischemia and reperfusion. Analysis of the temporal profile of the emission ratio during reperfusion using a double exponential function model can isolate kidneys very likely to fail when analysis by visual observation may be inconclusive.

\section{ACKNOWLEDGMENTS}

This work has been supported in part by funding from the Center for Biophotonics and the Lawrence Livermore National Laboratory through grant number (UCDRD Proposal LS03-009). The Center for Biophotonics, an NSF Science and Technology Center, is managed by the University of California, Davis, under Cooperative Agreement number PHY 0120999. This work was also supported in part by a research grant from the Transplant Hope Endowment, University of California, Davis, Medical Center, Sacramento. This work was performed in part at LLNL under the auspices of the US Department of Energy under contract W-7405-Eng-48 and in part under Contract DE-AC52-07NA27344.

\section{REFERENCES}

1. Carlsson, P. O., Kiuru, A., Nordin, A. et al, "Microdialysis measurements demonstrate a shift to nonoxidative glucose metabolism in rat pancreatic islets transplanted beneath the renal capsule," Surgery 132, 487-494 (2002)

2. Sola, A., Palacios, L., Lopez-Marti, J. et al, "Multiparametric monitoring of ischemia-reperfusion in rat kidney: Effect of preconditioning," Transplantation 75, 744-749 (2003)

3. Sommer, T. and Larsen, J. F. "Detection of intestinal ischemia using a microdialysis technique in an animal model," World Journal of Surgery 27, 416-420 (2003)

4. Chance, B. "Spectrophotometry of intracellular respiratory pigments," Science 120, 767-775 (1954)

5. Kobayashi, S., Nishiki, K., Kaede, K., and Ogata, E., "Optical consequences of blood substitution on tissue oxidation-reduction state microfluorometry," J Appl Phys 31, 93-96 (1971)

6. Mayevsky, A. and Chance, B., "Intracellular oxidation-reduction state measured in situ by a multichannel fiberoptic surface fluorometer," Science 217, 537-540 (1982)

7. Renault, G., Raynal, E., Sinet, M. et al, "In situ double-beam NADH laser fluorimetry: choice of a reference wavelength," Am J Physiol 246, H491-H499 (1984)

8. Mayevsky, A., Sonn, J., Luger-Hamer, M., and Nakache, R., "Real-time assessment of organ vitality during the transplantation procedure," Transplant Rev 17, 96-116 (2003)

9. Fitzgerald, J. T., Michalopoulou, A. P., Pivetti, C. D., Raman, R. N., Troppmann, C., and Demos, S. G., "Real-time assessment of in vivo renal ischemia using laser autofluorescence imaging," J Biom Opt 10, 044018 (2005)

10. Chance, B., Williamson, J. R., Jamieson, D., and Schoener, B., "Properties and kinetics of reduced pyridine nucleotide fluorescence of the isolated and in vivo rat heart" Biochemische Zeitschrift 341, 357-377 (1965)

11. Eklund, T., Wahlberg, G., Ungerstedt, U. et al, "Interstitial lactate, inosine, and hypoxanthine in rat kidney during normothermic ischaemia and recirculation," Acta Physiol Scand 143, 279-286 (1991)

12. Mehrabi, A., Golling, M., Busch, C. et al, "Experimental monitoring of hepatic metabolism by microdialysis glucose, lactate, and glutamate during surgical preparation of the liver hilus," J Surg Res 105, 128-135 (2002)

13. Jobsis, F. F., "Noninvasive, infrared monitoring of cerebral and myocardial oxygen sufficiency and circulatory parameters," Science 198, 1264-1267 (1977)

14. Simonson, S. G. and Piantadosi, C. A., "Near-infrared spectroscopy: Clinical applications," Crit Care Clin 12, 1019 (1996)

15. Chance, B., Cohen, P., Jobsis, F., and Schoener, B., "Intracellular oxidation-reduction states in vivo," Science 137, 499-508 (1962) 
16. Lin, H. J., Herman, P., and Lakowicz, J. R., "Fluorescence lifetime-resolved $\mathrm{pH}$ imaging of living cells," Cytometry 52A, 77-89 (2003)

17. Stratford, M. R. L., Parkins, C. S., Everett, S. A. et al, "Analysis of the acidic microenvironment in murine tumors by high-performance ion chromatography," Journal of Chromatography A 706, 459-462 (1995) 